Bull. Austral. Math. Soc.

Vol. 58 (1998) [43-56]

\title{
THE n-TH DERIVATIVE CHARACTERISATION OF MÖBIUS INVARIANT DIRICHLET SPACE
}

\author{
Rauno Aulaskari, Maria Nowak and Ruhan Zhao
}

In this paper we give the $n$-th derivative criterion for functions belonging to recently defined function spaces $Q_{p}$ and $Q_{p, 0}$. For a special parameter value $p=1$ this criterion is applied to BMOA and VMOA, and for $p>1$ it is applied to the Bloch space $\mathcal{B}$ and the little Bloch space $\mathcal{B}_{0}$. Further, a Carleson measure characterisation is given to $Q_{p}$, and in the last section the multiplier space from $H^{q}$ into $Q_{p}$ is considered.

\section{INTRODUCTION AND SOME AUXILIARY RESULTS}

Let $\mathcal{D}=\{z:|z|<1\}$ be the unit disk in the complex plane. Let $\varphi_{a}(z)=$ $(a-z) /(1-\bar{a} z)$ be a Möbius transformation of $\mathcal{D}$. An analytic function $f$ is said to be a Bloch function, denoted by $f \in \mathcal{B}$ (see [1]), if

$$
\sup _{z \in \mathcal{D}}\left(1-|z|^{2}\right)\left|f^{\prime}(z)\right|<\infty
$$

For $0<p<\infty$, we say that $f \in Q_{p}$ if $f$ is analytic and

$$
\sup _{a \in \mathcal{D}} \iint_{\mathcal{D}}\left|f^{\prime}(z)\right|^{2} g^{p}(z, a) d \sigma_{z}<\infty
$$

where $g(z, a)$ is the Green's function $\log |(1-\bar{a} z) /(z-a)|$ with logarithmic singularity at $a \in \mathcal{D}$ and $d \sigma_{z}$ is the usual area measure $d x d y$ on $\mathcal{D}$. These spaces were introduced by the first author and his collaborators and have been studied in [4], [6] and elsewhere. For $1<p<\infty$ the spaces $Q_{p}$ are all the same and equal to the Bloch space $\mathcal{B}$ (see [4, Theorem 1] and also [15, Corollary 2.4]). If $p=1$, we know by definition that $Q_{1}=$ BMOA (the space of analytic functions of bounded mean oscillation) [8]. For $0<p_{1}<p_{2} \leqslant 1$ we have $Q_{p_{1}} \subsetneq Q_{p_{2}} \subset$ BMOA (see [6, Theorem 2]). An important property that is common to these spaces $Q_{p}$ is that they are all invariant under Möbius transformations, that is, if $f \in Q_{p}$, then $f \circ \varphi_{a} \in Q_{p}$. This is well known in case of $\mathcal{B}$ and BMOA (see [2]). We note that in [10] a characterisation of boundary values

Received 24th November, 1997

Copyright Clearance Centre, Inc. Serial-fee code: 0004-9729/98 $\$ A 2.00+0.00$. 
for functions in $Q_{p}(0<p<1)$ is given. In this paper we shall derive a criterion for functions in $Q_{p}$ and $Q_{p, 0}$ in terms of their $n$-th derivatives. Further, we give a criterion for functions $f$ to belong to $Q_{p}$ and $Q_{p, 0}$ by $p$-Carleson measures. Also a sufficient condition for a function $f$ to belong to the multiplier space $\left(H^{q}, Q_{p}\right)$ is obtained. This last result should be compared with [11, Proposition 1 and Theorem 1]. First we need the following lemma:

LEMma 1. Let $f$ be an analytic function in $\mathcal{D}$. Then there exist positive constants $c_{1}, c_{2}$ such that

$$
\begin{aligned}
& c_{1}\left(\left|f^{(n)}(0)\right|^{2}+\iint_{\mathcal{D}}\left|f^{(n+1)}(z)\right|^{2}(1-|z|)^{\alpha+2} d \sigma_{z}\right) \\
& \quad \leqslant \iint_{\mathcal{D}}\left|f^{(n)}(z)\right|^{2}(1-|z|)^{\alpha} d \sigma_{z} \\
& \quad \leqslant c_{2}\left(\left|f^{(n)}(0)\right|^{2}+\iint_{\mathcal{D}}\left|f^{(n+1)}(z)\right|^{2}(1-|z|)^{\alpha+2} d \sigma_{z}\right)
\end{aligned}
$$

for $0<\alpha<\infty$.

Proof: Setting $f^{(n)}(z)=\sum_{n=0}^{\infty} a_{n} z^{n}$ we have $f^{(n+1)}(z)=\sum_{n=1}^{\infty} n a_{n} z^{n-1}$. Using Parseval's formula we get

$$
\begin{aligned}
& \iint_{\mathcal{D}}\left|f^{(n)}(z)\right|^{2}(1-|z|)^{\alpha} d \sigma_{z} \\
& \quad=2 \pi\left|f^{(n)}(0)\right|^{2} B(2, \alpha+1)+2 \pi \sum_{n=1}^{\infty}\left|a_{n}\right|^{2} B(2 n+2, \alpha+1)
\end{aligned}
$$

where we have the beta function $B(2 n+2, \alpha+1)=\int_{0}^{1} t^{2 n+1}(1-t)^{\alpha} d t$. On the other hand,

$$
\iint_{\mathcal{D}}\left|f^{(n+1)}(z)\right|^{2}(1-|z|)^{\alpha+2} d \sigma_{z}=2 \pi \sum_{n=1}^{\infty} n^{2}\left|a_{n}\right|^{2} B(2 n, \alpha+3) .
$$

By Stirling's formula $B(2 n+2, \alpha+1)=(\Gamma(2 n+2) \Gamma(\alpha+1)) /(\Gamma(2 n+\alpha+3)) \approx$ $1 /\left(n^{1+\alpha}\right)$ and $B(2 n, \alpha+3) \approx 1 /\left(n^{\alpha+3}\right)$. In the above, we use the notation $a \approx b$ to denote comparability of the quantities, that is, there are absolute positive constants $c_{1}, c_{2}$ satisfying $c_{1} b \leqslant a \leqslant c_{2} b$. Thus the assertion follows from (3) and (4).

We note that in the definition (1) of the space $Q_{p}$ the Green's function $g(z, a)$ can be replaced by $1-\left|\varphi_{a}(z)\right|^{2}$. Further, by [14, Lemma 3] we know that $f \in \mathcal{B}$ if and 
only if

$$
M_{f}^{n}=\sup _{z \in \mathcal{D}}\left(1-|z|^{2}\right)^{n}\left|f^{(n)}(z)\right|<\infty
$$

Using (2) and (5) and replacing $f(z)$ in (2) by $f_{a}(z)=f\left(\varphi_{a}(z)\right)-f(a)$ we get a criterion for functions in $Q_{p}$ :

Proposition. If $f$ is a Bloch function, then $f \in Q_{p}$ if and only if

$$
\sup _{a \in \mathcal{D}} \iint_{\mathcal{D}}\left|f_{a}^{(n)}(z)\right|^{2}(1-|z|)^{p+2(n-1)} d \sigma_{z}<\infty
$$

for $0<p<\infty$.

\section{The $n$-Th Derivative CRiteria For $Q_{p}$ AND $Q_{p, 0}$}

In this section we shall obtain the $n$-th derivative criteria for $Q_{p}$ and $Q_{p, 0}$. In case of the Bloch space $\mathcal{B}$ and the little Bloch space $\mathcal{B}_{0}$ corresponding criteria have been obtained by Axler [7] and Stroethoff [14]. Our results will generalise these to some other function spaces and, for example, for $p=1$ we have got the $n$-th derivative criterion for BMOA $\left(=Q_{1}\right)$ and VMOA $\left(=Q_{1,0}\right)$. The main result of this section is the following

Theorem 1. Let $n \geqslant 1$ and let $0<p<\infty$. Then, for an analytic function $f$ in $\mathcal{D}$, the following conditions are equivalent:

(i) $f \in Q_{p}$,

(ii) $\sup _{a \in \mathcal{D}} \iint_{\mathcal{D}}\left|f^{(n)}(z)\right|^{2}\left(1-\left|\varphi_{a}(z)\right|^{2}\right)^{p}\left(1-|z|^{2}\right)^{2 n-2} d \sigma_{z}<\infty$,

(iii) $\sup _{a \in \mathcal{D}} \iint_{\mathcal{D}}\left|f^{(n)}\left(\varphi_{a}(z)\right)\right|^{2}\left|\varphi_{a}^{\prime}(z)\right|^{2 n}\left(1-|z|^{2}\right)^{p+2 n-2} d \sigma_{z}<\infty$,

(iv) $\sup _{a \in \mathcal{D}} \iint_{\mathcal{D}}\left|f^{(n)}(z)\right|^{2} g^{p}(z, a)\left(1-|z|^{2}\right)^{2 n-2} d \sigma_{z}<\infty$.

Proof: By change of variables we have

$$
\begin{aligned}
\iint_{\mathcal{D}}\left|f^{(n)}(z)\right|^{2}\left(1-\left|\varphi_{a}(z)\right|^{2}\right)^{p}\left(1-|z|^{2}\right)^{2 n-2} d \sigma_{z} \\
\quad=\iint_{\mathcal{D}}\left|f^{(n)}\left(\varphi_{a}(z)\right)\right|^{2}\left|\varphi_{a}^{\prime}(z)\right|^{2 n}\left(1-|z|^{2}\right)^{p+2 n-2} d \sigma_{z}
\end{aligned}
$$

and thus (ii) is equivalent to (iii). Next we shall prove (i) is equivalent to (ii). 
(i) $\Longrightarrow$ (ii). We first consider the case $1<p \leqslant 2$. Constants appearing in the proofs denoted by $M$ are not always the same in each occurrence. Let $f \in Q_{p}=\mathcal{B}$. Then, by (5),

$$
\left|f^{(n)}\left(\varphi_{a}(z)\right)\right|\left(1-\left|\varphi_{a}(z)\right|^{2}\right)^{n}=\left|f^{(n)}\left(\varphi_{a}(z)\right)\right|\left|\varphi_{a}^{\prime}(z)\right|^{n}\left(1-|z|^{2}\right)^{n} \leqslant M_{f}^{n}
$$

and thus

$$
\begin{gathered}
\iint_{\mathcal{D}}\left|f^{(n)}\left(\varphi_{a}(z)\right)\right|^{2}\left|\varphi_{a}^{\prime}(z)\right|^{2 n}\left(1-|z|^{2}\right)^{p+2 n-2} d \sigma_{z} \\
\leqslant\left(M_{f}^{n}\right)^{2} \iint_{\mathcal{D}}\left(1-|z|^{2}\right)^{p-2} d \sigma_{z}=M<\infty
\end{gathered}
$$

for $1<p<\infty . \mathrm{By}(7)$ the assertion is true.

(ii) $\Longrightarrow$ (i). By $[\mathbf{1 4}$, Theorem 1(D)] we know that

$$
f \in \mathcal{B} \Longleftrightarrow \sup _{a \in \mathcal{D}} \iint_{\mathcal{D}}\left|f^{(n)}(z)\right|^{2}\left(1-\left|\varphi_{a}(z)\right|^{2}\right)^{2}\left(1-|z|^{2}\right)^{2 n-2} d \sigma_{z}<\infty
$$

Thus, by (8), we have settled the case $1<p \leqslant 2$.

Next we suppose $2<p<\infty$. By using (5) and [18, Lemma 4.2.2] the implication (i) $\Longrightarrow$ (ii) is trivial for these values of $p$. In the opposite direction the assertion is true since we have $\left(1-\left|\varphi_{a}(z)\right|^{2}\right)^{p}>\left(1-r^{2}\right)^{p}$ for $z \in \mathcal{D}(a, r)$, and thus

$$
\begin{aligned}
\infty & >\sup _{a \in \mathcal{D}} \iint_{\mathcal{D}}\left|f^{(n)}(z)\right|^{2}\left(1-\left|\varphi_{a}(z)\right|^{2}\right)^{p}\left(1-|z|^{2}\right)^{2 n-2} d \sigma_{z} \\
& \geqslant\left(1-r^{2}\right)^{p} \sup _{a \in \mathcal{D}} \iint_{\mathcal{D}(a, r)}\left|f^{(n)}(z)\right|^{2}\left(1-|z|^{2}\right)^{2 n-2} d \sigma_{z} .
\end{aligned}
$$

Hence, by [14, Theorem 1], $f \in \mathcal{B}=Q_{p}$ if (ii) is satisfied.

Finally we consider the case $0<p \leqslant 1$. For $n=1$ (i) $\Longrightarrow$ (ii) is true by [6, Proposition 1].

Suppose now that (i) $\Longrightarrow$ (ii) holds for some fixed $n$. We know that if $g$ is an analytic function in $\mathcal{D}$ then, by (2) in Lemma 1 ,

$$
\iint_{\mathcal{D}}\left|g^{\prime}(z)\right|^{2}\left(1-|z|^{2}\right)^{\alpha+2} d \sigma_{z} \leqslant M \iint_{\mathcal{D}}|g(z)|^{2}\left(1-|z|^{2}\right)^{\alpha} d \sigma_{z}
$$


for $0<\alpha<\infty$. If we apply (9) to the function $g(z)=f^{(n)}(z) /(1-\bar{a} z)^{p}$ and $\alpha=$ $2 n-2+p$ and multiply both sides of the inequality (9) by $\left(1-|a|^{2}\right)^{p}$ we obtain

$$
\begin{aligned}
& \iint_{\mathcal{D}}\left(\frac{\left|f^{(n+1)}(z)\right|^{2}}{|1-\bar{a} z|^{2 p}}+\frac{p^{2}|a|^{2}\left|f^{(n)}(z)\right|^{2}}{|1-\bar{a} z|^{2 p+2}}\right. \\
& \left.\quad+2 \operatorname{Re} \frac{p a f^{(n+1)}(z) \overline{f^{(n)}(z)}}{(1-\bar{a} z)^{p}(1-a \bar{z})^{p+1}}\right)\left(1-|z|^{2}\right)^{2 n+p}\left(1-|a|^{2}\right)^{p} d \sigma_{z} \\
& \leqslant M \iint_{\mathcal{D}}\left|f^{(n)}(z)\right|^{2} \frac{\left(1-|a|^{2}\right)^{p}\left(1-|z|^{2}\right)^{p+2 n-2}}{|1-\bar{a} z|^{2 p}} d \sigma_{z} \\
& =M \iint_{\mathcal{D}}\left|f^{(n)}(z)\right|^{2}\left(1-\left|\varphi_{a}(z)\right|^{2}\right)^{p}\left(1-|z|^{2}\right)^{2 n-2} d \sigma_{z} .
\end{aligned}
$$

By the assumption we know that in (10) the supremum of the upper bound is finite. Since $f \in \mathcal{B}$, we get by using (5) and [18, Lemma 4.2.2]

$$
\begin{aligned}
& \iint_{\mathcal{D}} \frac{\left|f^{(n)}(z) f^{(n+1)}(z)\right|}{|1-\bar{a} z|^{2 p+1}}\left(1-|z|^{2}\right)^{2 n+p}\left(1-|a|^{2}\right)^{p} d \sigma_{z} \\
& \quad \leqslant M_{f}^{n} M_{f}^{n+1} \iint_{\mathcal{D}} \frac{\left(1-|z|^{2}\right)^{p-1}\left(1-|a|^{2}\right)^{p}}{|1-\bar{a} z|^{2 p+1}} d \sigma_{z} \leqslant M<\infty
\end{aligned}
$$

for all $a \in \mathcal{D}$. Moreover,

$$
\begin{aligned}
& \iint_{\mathcal{D}} \frac{\left|f^{(n)}(z)\right|^{2}}{|1-\bar{a} z|^{2 p+2}}\left(1-|z|^{2}\right)^{2 n+p}\left(1-|a|^{2}\right)^{p} d \sigma_{z} \\
& \quad \leqslant 4 \iint_{\mathcal{D}}\left|f^{(n)}(z)\right|^{2} \frac{\left(1-|z|^{2}\right)^{p+2 n-2}\left(1-|a|^{2}\right)^{p}}{|1-\bar{a} z|^{2 p}} d \sigma_{z} \\
& \quad=4 \iint_{\mathcal{D}}\left|f^{(n)}(z)\right|^{2}\left(1-\left|\varphi_{a}(z)\right|^{2}\right)^{p}\left(1-|z|^{2}\right)^{2 n-2} d \sigma_{z}
\end{aligned}
$$

and again the upper bound is finite. Hence, in view of (10), if $f \in Q_{p}$ (even, in fact, if $f \in \mathcal{B})$ then

$$
\sup _{a \in \mathcal{D}} \iint_{\mathcal{D}}\left|f^{(n)}(z)\right|^{2}\left(1-\left|\varphi_{a}(z)\right|^{2}\right)^{p}\left(1-|z|^{2}\right)^{2 n-2} d \sigma_{z}<\infty
$$


48

R. Aulaskari, M. Nowak and R. Zhao

[6]

implies

$$
\sup _{a \in \mathcal{D}} \iint_{\mathcal{D}}\left|f^{(n+1)}(z)\right|^{2}\left(1-\left|\varphi_{a}(z)\right|^{2}\right)^{p}\left(1-|z|^{2}\right)^{2 n} d \sigma_{z}<\infty .
$$

Thus, by induction on $n$, (i) implies (ii) for all $n \geqslant 1$.

(ii) $\Longrightarrow$ (i). In this case we shall also proceed by induction. If (ii) holds for $0<p \leqslant 1$, then by [14, Theorem 1] $f \in \mathcal{B}$. For $n=1$ the implication is true by [6, Proposition 1]. Suppose now that (ii) $\Longrightarrow$ (i) is true for some fixed $n$. By Lemma 1 we have for an analytic function $g$ in $\mathcal{D}$,

$$
\iint_{\mathcal{D}}|g(z)|^{2}\left(1-|z|^{2}\right)^{\alpha} d \sigma_{z} \leqslant M\left(|g(0)|^{2}+\iint_{\mathcal{D}}\left|g^{\prime}(z)\right|^{2}\left(1-|z|^{2}\right)^{\alpha+2} d \sigma_{z}\right),
$$

where $0<\alpha<\infty$. Assume that

$$
\sup _{a \in \mathcal{D}} \iint_{\mathcal{D}}\left|f^{(n+1)}(z)\right|^{2}\left(1-\left|\varphi_{a}(z)\right|^{2}\right)^{p}\left(1-|z|^{2}\right)^{2 n} d \sigma_{z}<\infty .
$$

In (11) we substitute $g(z)=\left(f^{(n)}(z)\right) /(1-\bar{a} z)^{p}, \alpha=2 n-2+p$ and multiply both sides of $(11)$ by $\left(1-|a|^{2}\right)^{p}$. Then we get

$$
\begin{aligned}
& \iint_{\mathcal{D}}\left|f^{(n)}(z)\right|^{2}\left(1-\left|\varphi_{a}(z)\right|^{2}\right)^{p}\left(1-|z|^{2}\right)^{2 n-2} d \sigma_{z} \\
& =\iint_{\mathcal{D}}\left|\frac{f^{(n)}(z)}{(1-\bar{a} z)^{p}}\right|^{2}\left(1-|z|^{2}\right)^{2 n-2+p}\left(1-|a|^{2}\right)^{p} d \sigma_{z} \\
& \leqslant M\left(\left|f^{(n)}(0)\right|^{2}\left(1-|a|^{2}\right)^{p}+\iint\left|\frac{d}{d z} \frac{f^{(n)}(z)}{(1-\bar{a} z)^{p}}\right|^{2}\left(1-|z|^{2}\right)^{2 n+p}\left(1-|a|^{2}\right)^{p} d \sigma_{z}\right) \\
& =M\left(\left|f^{(n)}(0)\right|^{2}\left(1-|a|^{2}\right)^{p}\right. \\
& \quad+\iint_{\mathcal{D}} \mid \frac{f^{(n+1)}(z)}{(1-\bar{a} z)^{p}+p \bar{a}} \frac{f^{(n)}(z)}{\left.\left.(1-\bar{a} z)^{p+1}\right|^{2}\left(1-|z|^{2}\right)^{2 n+p}\left(1-|a|^{2}\right)^{p} d \sigma_{z}\right)} \\
& \leqslant \quad\left(\left|f^{(n)}(0)\right|^{2}\left(1-|a|^{2}\right)^{p}+\iint_{\mathcal{D}} \frac{\left|f^{(n+1)}(z)\right|^{2}}{|1-\bar{a} z|^{2 p}}\left(1-|z|^{2}\right)^{2 n+p}\left(1-|a|^{2}\right)^{p} d \sigma_{z}\right. \\
& \quad+p^{2}|a|^{2} \iint_{\mathcal{D}} \frac{\left|f^{(n)}(z)\right|^{2}}{|1-\bar{a} z|^{2 p+2}}\left(1-|z|^{2}\right)^{2 n+p}\left(1-|a|^{2}\right)^{p} d \sigma_{z} \\
& \left.\quad+2 p|a| \iint_{\mathcal{D}} \frac{\left|f^{(n+1)}(z) f^{(n)}(z)\right|}{|1-\bar{a} z|^{2 p+1}}\left(1-|z|^{2}\right)^{2 n+p}\left(1-|a|^{2}\right)^{p} d \sigma_{z}\right) .
\end{aligned}
$$

https://doi.org/10.1017/S0004972700031993 Published online by Cambridge University Press 
Since $f \in \mathcal{B}$, we have $\left|f^{(n)}(0)\right|^{2}\left(1-|a|^{2}\right)^{p} \leqslant\left(M_{f}^{n}\right)^{2}$. By assumption,

$$
\begin{aligned}
& \iint_{\mathcal{D}} \frac{\left|f^{(n+1)}(z)\right|^{2}}{|1-\bar{a} z|^{2 p}}\left(1-|z|^{2}\right)^{2 n+p}\left(1-|a|^{2}\right)^{p} d \sigma_{z} \\
& \quad=\iint_{\mathcal{D}}\left|f^{(n+1)}(z)\right|^{2}\left(1-\left|\varphi_{a}(z)\right|^{2}\right)^{p}\left(1-|z|^{2}\right)^{2 n} d \sigma_{z} \leqslant M<\infty .
\end{aligned}
$$

The other terms involving integrals can be estimated as follows (see [18, Lemma 4.2.2]):

$$
\begin{gathered}
\iint_{\mathcal{D}} \frac{\left|f^{(n)}(z)\right|^{2}}{|1-\bar{a} z|^{2 p+2}}\left(1-|z|^{2}\right)^{2 n+p}\left(1-|a|^{2}\right)^{p} d \sigma_{z} \\
\quad \leqslant\left(M_{f}^{n}\right)^{2}\left(1-|a|^{2}\right)^{p} \iint_{\mathcal{D}} \frac{\left(1-|z|^{2}\right)^{p}}{|1-\bar{a} z|^{2 p+2}} d \sigma_{z} \\
\quad \leqslant M\left(M_{f}^{n}\right)^{2}\left(1-|a|^{2}\right)^{p} \frac{1}{\left(1-|a|^{2}\right)^{p}} \leqslant M
\end{gathered}
$$

and

$$
\begin{gathered}
\iint_{\mathcal{D}} \frac{\left|f^{(n+1)}(z) f^{(n)}(z)\right|}{|1-\bar{a} z|^{2 p+1}}\left(1-|z|^{2}\right)^{2 n+p}\left(1-|a|^{2}\right)^{p} d \sigma_{z} \\
\leqslant M_{f}^{n} M_{f}^{n+1}\left(1-|a|^{2}\right)^{p} \iint_{\mathcal{D}} \frac{\left(1-|z|^{2}\right)^{p-1}}{|1-\bar{a} z|^{2 p+1}} d \sigma_{z} \\
\leqslant M M_{f}^{n} M_{f}^{n+1}\left(1-|a|^{2}\right)^{p} \frac{1}{\left(1-|a|^{2}\right)^{p}} \leqslant M .
\end{gathered}
$$

By assumption we have $f \in Q_{p}$ and thus, by induction, we have proved (ii) $\Longrightarrow$ (i).

(iv) $\Longrightarrow$ (ii). This is obvious from the inequality $1-\left|\varphi_{a}(z)\right|^{2} \leqslant 2 g(z, a)$ for all $z, a \in \mathcal{D}$.

(ii) $\Longrightarrow$ (iv). Let

$$
\begin{aligned}
I(a)= & \iint_{\mathcal{D}}\left|f^{(n)}(z)\right|^{2} g^{\mathcal{p}}(z, a)\left(1-|z|^{2}\right)^{2 n-2} d \sigma_{z} \\
= & \iint_{\mathcal{D}(a, 1 / 4)}\left|f^{(n)}(z)\right|^{2} g^{p}(z, a)\left(1-|z|^{2}\right)^{2 n-2} d \sigma_{z} \\
& \quad+\iint_{\mathcal{D} \backslash \mathcal{D}(a, 1 / 4)}\left|f^{(n)}(z)\right|^{2} g^{\mathcal{p}}(z, a)\left(1-|z|^{2}\right)^{2 n-2} d \sigma_{z}=I_{1}(a)+I_{2}(a),
\end{aligned}
$$


where $\mathcal{D}(a, 1 / 4)=\left\{z \in \mathcal{D}|| \varphi_{a}(z) \mid<1 / 4\right\}$. Since

$$
g(z, a)=\log \frac{1}{\left|\varphi_{a}(z)\right|} \begin{cases}\geqslant \log 4>1, & z \in \mathcal{D}(a, 1 / 4), \\ \leqslant 4\left(1-\left|\varphi_{a}(z)\right|^{2}\right), & z \in \mathcal{D} \backslash \mathcal{D}(a, 1 / 4),\end{cases}
$$

we obtain, for $p_{0}=\max (p, 2)$, that

$$
I_{1}(a) \leqslant \iint_{\mathcal{D}(a, 1 / 4)}\left|f^{(n)}(z)\right|^{2} g^{p_{0}}(z, a)\left(1-|z|^{2}\right)^{2 n-2} d \sigma_{z}
$$

and

$$
I_{2}(a) \leqslant 4^{p} \iint_{\mathcal{D} \backslash \mathcal{D}(a, 1 / 4)}\left|f^{(n)}(z)\right|^{2}\left(1-\left|\varphi_{a}(z)\right|^{2}\right)^{p}\left(1-|z|^{2}\right)^{2 n-2} d \sigma_{z} .
$$

Since we have proved that (ii) implies $f \in Q_{p} \subset \mathcal{B}$, we get that (5) is satisfied, and so, from $p_{0} \geqslant 2$, we get

$$
\begin{aligned}
\sup _{a \in \mathcal{D}} I_{1}(a) & \leqslant\left(M_{f}^{n}\right)^{2} \sup _{a \in \mathcal{D}} \iint_{\mathcal{D}(a, 1 / 4)} g^{p_{0}}(z, a)\left(1-|z|^{2}\right)^{-2} d \sigma_{z} \\
& =\left(M_{f}^{n}\right)^{2} \iint_{\mathcal{D}(0,1 / 4)}\left(\log \frac{1}{|w|}\right)^{p_{0}}\left(1-|w|^{2}\right)^{-2} d \sigma_{w}<\infty .
\end{aligned}
$$

By (ii), $\sup _{a \in \mathcal{D}} I_{2}(a)<\infty$. Thus

$$
\sup _{a \in \mathcal{D}} I(a)=\sup _{a \in \mathcal{D}}\left(I_{1}(a)+I_{2}(a)\right)<\infty
$$

or (iv) is satisfied. The proof is completed.

Contained in the Bloch space is the little Bloch space $\mathcal{B}_{0}$, which is by definition the set of all analytic functions $f$ in $\mathcal{D}$ for which $\left(1-|z|^{2}\right)\left|f^{\prime}(z)\right| \rightarrow 0$ as $|z| \rightarrow 1$. For $0<p<\infty$, we say that $f \in Q_{p, 0}$ if $f$ is analytic and

$$
\lim _{|a| \rightarrow 1} \iint_{\mathcal{D}}\left|f^{\prime}(z)\right|^{2} g^{p}(z, a) d \sigma_{z}=0
$$

By [4, Corollary 2] we know that $Q_{p, 0}=\mathcal{B}_{0}$ for $1<p<\infty$ (see also [16]). On the other hand, if $p=1$ we have that $Q_{p, 0}=$ VMOA (the space of analytic functions of vanishing mean oscillation) [12]. If $0<p_{1}<p_{2} \leqslant 1$, then $Q_{p_{1}, 0} \subsetneq Q_{p_{2}, 0}$ (see [6]). By the above proof, Theorem 2 and $[14$, Lemma 4] we get the corresponding theorem in the limit case: 
THEorem 2. Let $n \geqslant 1$ and let $0<p<\infty$. Then, for an analytic function $f$ in $\mathcal{D}$, the following conditions are equivalent:

$$
\begin{aligned}
& \text { (i) } f \in Q_{p, 0}, \\
& \text { (ii) } \lim _{|a| \rightarrow 1} \iint_{\mathcal{D}}\left|f^{(n)}(z)\right|^{2}\left(1-\left|\varphi_{a}(z)\right|^{2}\right)^{p}\left(1-|z|^{2}\right)^{2 n-2} d \sigma_{z}=0, \\
& \text { (iii) }\left.\lim _{|a| \rightarrow 1} \iint_{\mathcal{D}} \int f^{(n)}\left(\varphi_{a}(z)\right)\right|^{2}\left|\varphi_{a}^{\prime}(z)\right|^{2 n}\left(1-|z|^{2}\right)^{p+2 n-2} d \sigma_{z}=0, \\
& \text { (iv) } \lim _{|a| \rightarrow 1} \iint\left|f^{(n)}(z)\right|^{2} g^{p}(z, a)\left(1-|z|^{2}\right)^{2 n-2} d \sigma_{z}=0 .
\end{aligned}
$$

\section{3. $Q_{p}$ AND ENTIRE FUNCTIONS}

In this section we shall generalise Theorem 1 by replacing the weight factor by an infinite series of weight factors. For $1<p<\infty$ and $n=1$ this case was considered in [3] when criteria for the Bloch space were established.

Theorem 3. Let $0<p<\infty$, let $n \geqslant 1$ be an integer, and let $E(\rho)=\sum_{k=0}^{\infty} b_{k} \rho^{k}$ be an entire function with $b_{k} \geqslant 0$ and $b_{0}>0$. If

$$
\varlimsup_{k \rightarrow \infty} k \sqrt[k]{b_{k}}<2 e
$$

then, for an analytic function $f$ in $\mathcal{D}$, the following conditions are equivalent:

(i) $f \in Q_{p}$,

(ii) $\sup _{a \in \mathcal{D}} \iint_{\mathcal{D}}\left|f^{(n)}(z)\right|^{2}\left(1-|z|^{2}\right)^{2 n-2} g^{p}(z, a) E(g(z, a)) d \sigma_{z}<\infty$,

(iii) $\sup _{a \in \mathcal{D}} \iint_{\mathcal{D}}\left|f^{(n)}(z)\right|^{2}\left(1-|z|^{2}\right)^{2 n-2}\left(1-\left|\varphi_{a}(z)\right|^{2}\right)^{p} E(g(z, a)) d \sigma_{z}<\infty$.

Proof: (i) $\Longrightarrow$ (ii). Let $E_{1}(\rho)=E(\rho)-b_{0}=\sum_{k=1}^{\infty} b_{k} \rho^{k}$. Since $f \in Q_{p}$, we get by Theorem 1,

$$
b_{0} \sup _{a \in \mathcal{D}} \iint_{\mathcal{D}}\left|f^{(n)}(z)\right|^{2}\left(1-|z|^{2}\right)^{2 n-2} g^{\mathcal{P}}(z, a) d \sigma_{z}<\infty .
$$

Since $f \in Q_{p} \subset \mathcal{B}$, we have by (5),

$$
M_{f}^{n}=\sup _{z \in \mathcal{D}}\left|f^{(n)}(z)\right|\left(1-|z|^{2}\right)^{n}<\infty .
$$


Thus

$$
\begin{aligned}
& \sup _{a \in \mathcal{D}} \iint_{\mathcal{D}}\left|f^{(n)}(z)\right|^{2}\left(1-|z|^{2}\right)^{2 n-2} g^{p}(z, a) E_{1}(g(z, a)) d \sigma_{z} \\
& \quad \leqslant \sum_{k=1}^{\infty} b_{k}\left(M_{f}^{n}\right)^{2} \sup _{a \in \mathcal{D}} \iint_{\mathcal{D}}\left(1-|z|^{2}\right)^{-2} g^{k+p}(z, a) d \sigma_{z} \\
& \quad=\sum_{k=1}^{\infty} b_{k}\left(M_{f}^{n}\right)^{2} \iint_{\mathcal{D}}\left(1-|w|^{2}\right)^{-2}\left(\log \frac{1}{|w|}\right)^{k+p} d \sigma_{w} \\
& =\left(M_{f}^{n}\right)^{2} \sum_{k=1}^{\infty} b_{k} J(k+p),
\end{aligned}
$$

where $J(k+p)=\iint_{\mathcal{D}}\left(1-|w|^{2}\right)^{-2}(\log 1 /|w|)^{k+p} d \sigma_{w}$. By [17, Lemma 3.3] we see that (12) implies $\sum_{k=1}^{\infty} b_{k} J(k+p)<\infty$. Thus

$$
\sup _{a \in \mathcal{D}} \iint_{\mathcal{D}}\left|f^{(n)}(z)\right|^{2}\left(1-|z|^{2}\right)^{2 n-2} g^{p}(z, a) E_{1}(g(z, a)) d \sigma_{z}<\infty .
$$

Combining (13) and (14) we see that (ii) is true.

(ii) $\Longrightarrow$ (iii). This is obvious by $1-\left|\varphi_{a}(z)\right|^{2} \leqslant 2 g(z, a)$ for $z, a \in \mathcal{D}$.

(iii) $\Longrightarrow$ (i). Since $b_{0}>0$ we get from (iii) that

$$
b_{0} \sup _{a \in \mathcal{D}} \iint_{\mathcal{D}}\left|f^{(n)}(z)\right|^{2}\left(1-|z|^{2}\right)^{2 n-2}\left(1-\left|\varphi_{a}(z)\right|^{2}\right)^{p} d \sigma_{z}<\infty
$$

and so, by Theorem 1, we see that $f \in Q_{p}$.

Theorem 3 is critical in the following sense:

TheOREM 4. Let $0<p<\infty$, let $n \geqslant 1$ be an integer, and let $E(\rho)=\sum_{k=0}^{\infty} b_{k} \rho^{k}$ be an entire function with $b_{k} \geqslant 0$ and $b_{0}>0$. Suppose that

$$
\varlimsup_{k \rightarrow \infty} k \sqrt[k]{b_{k}}>2 e
$$

and for an analytic function $f$ on $\mathcal{D}$, one of the following conditions is satisfied:

$$
\begin{aligned}
& \text { (i) } \sup _{a \in \mathcal{D}} \iint_{\mathcal{D}}\left|f^{(n)}(z)\right|^{2}\left(1-|z|^{2}\right)^{2 n-2} g^{p}(z, a) E(g(z, a)) d \sigma_{z}<\infty, \\
& \text { (ii) } \sup _{a \in \mathcal{D}} \iint_{\mathcal{D}}\left|f^{(n)}(z)\right|^{2}\left(1-|z|^{2}\right)^{2 n-2}\left(1-\left|\varphi_{a}(z)\right|^{2}\right)^{p} E(g(z, a)) d \sigma_{z}<\infty .
\end{aligned}
$$


Then $f$ is a polynomial, whose degree is less than $n$, or a constant.

We need the following lemma which can be proved in much the same way as in [17, Lemma 2.9].

Lemma 2. Let $0<p<\infty$, let $0<r<1$, and let $n \geqslant 1$ be an integer. Then, for an analytic function $f$ on $\mathcal{D}$ and $a \in \mathcal{D}$,

$$
\left|f^{(n)}(a)\right|\left(1-|a|^{2}\right)^{n} \leqslant \frac{16}{\pi c(n) r^{2}(\log 1 / r)^{p}} \iint_{\mathcal{D}}\left|f^{(n)}(z)\right|^{2}\left(1-|z|^{2}\right)^{2 n-2} g^{p}(z, a) d \sigma_{z},
$$

where $c(n)$ is a constant depending only on $n$.

By means of Lemma 2, the proof of Theorem 4 is same as in [17, Theorem 3.10]. We omit it here.

\section{4. $Q_{p}$ and Carleson measure}

Let $I$ be a subarc on the unit circle and let

$$
S(I)=\{z: z /|z| \in I, 1-|I| \leqslant|z|<1\},
$$

where $|I|$ denotes the arc length of $I$. A positive measure $\mu$ on $\mathcal{D}$ is a bounded $p$-Carleson measure, $0<p<\infty$, if

$$
\mu(S(I))=O\left(|I|^{p}\right) .
$$

If the right-hand side of $(15)$ is $o\left(|I|^{p}\right)$ then we say that $\mu$ is a compact $p$-Carleson measure.

It has been proved by Stegenga [13] (see also [9] for the case of the unit ball in $\mathbb{C}^{n}$ ) that, for $1 \leqslant p<\infty, \mu$ is a bounded $p$-Carleson measure if and only if

$$
\iint_{\mathcal{D}}|f(z)|^{2} d \mu(z) \leqslant C\left(\iint_{\mathcal{D}}\left|f^{\prime}(z)\right|^{2}\left(1-|z|^{2}\right)^{p} d \sigma_{z}+|f(0)|^{2}\right)
$$

for all analytic functions $f$ on $\mathcal{D}$ for which the integral on the right-hand side of the inequality (16) is finite.

If $0<p<1$ and inequality (16) holds then $\mu$ is a bounded $p$-Carleson measure. However, in this case the implication in the opposite direction is not true [13].

In view of [5, Lemma 2.1] Theorems 1 and 2 give immediately

Theorem 5. Let $n \geqslant 1$ be a natural integer and let $0<p<\infty$. Then, for an analytic function $f$ in $\mathcal{D}$, we have

$$
f \in Q_{p} \text { if and only if } d \mu=\left|f^{(n)}(z)\right|^{2}\left(1-|z|^{2}\right)^{2 n-2+p} d \sigma_{z}
$$
is a bounded $p$-Carleson measure, $f \in Q_{p, 0}$ if and only if $d \mu=\left|f^{(n)}(z)\right|^{2}\left(1-|z|^{2}\right)^{2 n-2+p} d \sigma_{z}$ is a compact p-Carleson measure. 


\section{A SUFFICIENT CONDITION FOR MULTIPLIERS FROM $H^{q}$ INTO $Q_{p}$}

For $0<q \leqslant \infty$, by $H^{q}$ we denote the space of functions $f$, analytic in $\mathcal{D}$, for which

$$
M_{q}^{q}(r, f)=\frac{1}{2 \pi} \int_{0}^{2 \pi}\left|f\left(r e^{i \theta}\right)\right|^{q} d \theta
$$

or

$$
M_{\infty}(r, f)=\max _{0 \leqslant \theta<2 \pi}\left|f\left(r e^{i \theta}\right)\right|
$$

remains bounded as $r \rightarrow 1$.

Let $A$ and $B$ be two vector spaces of sequences. A sequence $\lambda=\left\{\lambda_{n}\right\}$ is said to be a multiplier from $A$ to $B$ if $\left\{\lambda_{n} \alpha_{n}\right\} \in B$ whenever $\left\{\alpha_{n}\right\} \in A$. The set of all multipliers from $A$ to $B$ will be denoted by $(A, B)$. In this section we regard spaces of analytic functions in $\mathcal{D}$ as sequence spaces by identifying a function with its sequence of Taylor coefficients.

From [11, Proposition 1] we get the following result:

THEOREM MP. If $1<p \leqslant 2$ then a necessary and sufficient condition that $g \in$ $\left(H^{p}, \mathrm{BMOA}\right)$ is that

$$
M_{q}\left(r, g^{\prime}\right) \leqslant c /(1-r), 0<r<1,
$$

where $1 / p+1 / q=1$ and $\left(H^{1}, \mathrm{BMOA}\right)=\mathcal{B}$.

We shall need the multiplier transformation $D^{s} g$ of $g, g(z)=\sum_{n=0}^{\infty} \widehat{g}(n) z^{n}$, which is defined by

$$
D^{s} g(z)=\sum_{n=0}^{\infty}(n+1)^{s} \widehat{g}(n) z^{n}, s \text { any real number. }
$$

Now we are ready to prove

Theorem 6. If $1 \leqslant q \leqslant 2,0<p \leqslant 1$ then a sufficient condition that $g \in$ $\left(H^{q}, Q_{p}\right)$ is that

$$
M_{q^{\prime}}\left(r, g^{\prime}\right)=\left(\frac{1}{2 \pi} \int_{0}^{2 \pi}\left|g^{\prime}\left(r e^{i \theta}\right)\right|^{q^{\prime}} d \theta\right)^{1 / q^{\prime}} \leqslant \frac{c}{(1-r)^{(1+p) / 2}}
$$

where $1 / q+1 / q^{\prime}=1$. In particular, $g \in\left(H^{1}, Q_{p}\right)$ if

$$
M_{\infty}\left(g^{\prime}, r\right)=\max _{|z|=r}\left|g^{\prime}(z)\right| \leqslant \frac{c}{(1-r)^{(1+p) / 2}} .
$$

Proof: Let $f \in H^{q}$ and let

$$
h(z)=f \star g(z)=\sum_{n=0}^{\infty} \widehat{f}(n) \widehat{g}(n) z^{n} .
$$


Then

$$
\begin{aligned}
& \left|r^{2} D^{2} h\left(r^{2} e^{i t}\right)\right|^{2}=\left|\frac{1}{2 \pi} \int_{0}^{2 \pi} D^{1} f\left(r e^{i \theta}\right) D^{1} g\left(r e^{i(t-\theta)}\right) d \theta\right|^{2} \\
& \leqslant M_{q^{\prime}}^{2}\left(r, g^{\prime}\right) M_{q}^{2}\left(r, f^{\prime}\right) \leqslant \frac{c}{(1-r)^{1+p}} M_{q}^{2}\left(r, f^{\prime}\right) .
\end{aligned}
$$

Hence, by [11, Lemma 1],

$$
(1-r)^{2+p} M_{\infty}^{2}\left(r^{2}, h^{\prime \prime}\right) \leqslant c(1-r) M_{q}^{2}\left(r, f^{\prime}\right)
$$

and, by Lemma HL1 in [11],

$$
\int_{0}^{1}(1-r)^{2+p} M_{\infty}^{2}\left(r^{2}, h^{\prime \prime}\right) d r \leqslant c \int_{0}^{1}(1-r) M_{q}^{2}\left(r, f^{\prime}\right) d r<\infty .
$$

We shall show that if

$$
\int_{0}^{1}(1-r)^{2+p} M_{\infty}^{2}\left(r^{2}, h^{\prime \prime}\right) d r<\infty
$$

then $h \in Q_{p}$.

By [6, Lemma 4], we have

$$
\sup _{a \in \mathcal{D}} \int_{0}^{2 \pi} \frac{\left(1-|a|^{2}\right)^{p}}{\left|1-\bar{a} r e^{i t}\right|^{2 p}} d t<\infty
$$

and thus

$$
\begin{aligned}
& \sup _{a \in \mathcal{D}} \int_{\mathcal{D}}\left|h^{\prime \prime}(z)\right|^{2}\left(1-|z|^{2}\right)^{p+2} \frac{\left(1-|a|^{2}\right)^{p}}{|1-\bar{a} z|^{2 p}} d \sigma_{z} \\
& \quad \leqslant \sup _{a \in \mathcal{D}} \int_{0}^{1}(1-r)^{2+p} M_{\infty}^{2}\left(r, h^{\prime \prime}\right) \int_{0}^{2 \pi} \frac{\left(1-|a|^{2}\right)^{p}}{\left|1-\bar{a} r e^{i t}\right|^{2 p}} d t d r \\
& \quad \leqslant c \int_{0}^{1}(1-r)^{2+p} M_{\infty}^{2}\left(r, h^{\prime \prime}\right) d r<\infty
\end{aligned}
$$

which, by our Theorem 1, implies $h \in Q_{p}$ for $n=2$.

\section{REFERENCES}

[1] J. M. Anderson, J. Clunie and Ch. Pommerenke, 'On Bloch functions and normal functions', J. Reine Angew. Math. 240 (1974), 12-37.

[2] J. Arazy, S.D. Fisher and J. Peetre, 'Möbius invariant function spaces', J. Reine Angew. Math. 363 (1985), 110-145. 
[3] R. Aulaskari, Y. He and R. Zhao, 'On entire functions, Bloch and normal functions', Chinese Ann. Math. Ser. B 17 (1996), 139-148.

[4] R. Aulaskari and P. Lappan, 'Criteria for an analytic function to be Bloch and a harmonic or meromorphic function to be normal', in Complex analysis and its applications, Pitman Research Notes in Mathematics Series 305 (Longman Scientific and Technical, Harlow, 1994), pp. 136-146.

[5] R. Aulaskari, D. A. Stegenga and J. Xiao, 'Some subclasses of BMOA and their characterization in terms of Carleson measures', Rocky Mountain J. Math. 26 (1996), 485-506.

[6] R. Aulaskari, J. Xiao and R. Zhao, 'On subspaces and subsets of BMOA and UBC', Analysis 15 (1995), 101-121.

[7] S. Axler, 'The Bergman space, the Bloch space and commutators of multiplication operators', Duke Math. J. 53 (1986), 315-332.

[8] A. Baernstein II, 'Analytic functions of bounded mean oscillation', in Aspects of contemporary complex analysis (Academic Press, London, New York, 1980), pp. 2-26.

[9] J.A. Cima and W. Wogen, 'A Carleson measure theorem for the Bergman space on the ball', J. Operator Theory 7 (1982), 157-165.

[10] M. Essén and J. Xiao, 'Some results on $Q_{p}$ spaces, $0<p<1$ ', J. Reine Angew. Math. 485 (1997), 173-195.

[11] M. Mateljevic and M. Pavlovic, 'Multipliers of $H^{p}$ and BMOA', Pacific J. Math. 146 (1990), 71-84.

[12] D. Sarason, 'Functions of vanishing mean oscillation', Trans. Amer. Math. Soc. 205 (1975), 391-405.

[13] D.A. Stegenga, 'Multipliers of Dirichlet space', Illinois J. Math. 24 (1980), 113-139.

[14] K. Stroethoff, 'Besov-type characterizations for the Bloch space', Bull. Austral. Math. Soc. 39 (1989), 405-420.

[15] J. Xiao, 'Carleson measure, atomic decomposition and free interpolation from Bloch space', Ann. Acad. Sci. Fenn. Ser. A I Math. 19 (1994), 35-46.

[16] J. Xiao and L. Zhong, 'On little Bloch space, atomic decomposition and free interpolation', Complex Variables Theory Appl. 27 (1995), 175-184.

[17] R. Zhao, 'On a general family of function spaces', Ann. Acad. Sci. Fenn. Math. Dissertationes 105 (1996), 1-56.

[18] K.H. Zhu, Operator Theory in Function Spaces (Marcel Dekker, Inc., New York and Basel, 1990).

University of Joensuu

PO Box 111

FIN-8010 Joensuu

Finland

e-mail: aulaskari@joyl.joensuu.fi

University of Joensuu

PO Box 111

FIN-8010 Joensuu

Finland
Institute of Mathematics

Maria Curie-Sklodowska University

pl. M. Curie-Skodowskiej 1

20-031 Lublin

Poland 